\title{
Respuestas pos-sísmicas con un enfoque del desarrollo humano y de derechos humanos: (in)visibilizar actores, derechos y capacidades
}

Johannes M. Waldmueller,* Nelson Nogales, **

Ryan Cobey,*** María Carolina Urigüen****

\section{RESUMEN}

Basándose en el marco de los indicadores de derechos humanos para la política pública, el presente artículo brinda un análisis de la perspectiva fiscalista empleada por el gobierno de Ecuador después del terremoto del 16 de abril de 2016, para administrar y gestionar el desastre natural y la reconstrucción. Dicha perspectiva conllevó a la vulneración de algunos derechos humanos, afectó las capacidades de resiliencia de la población, y dificultó la generación de condiciones adecuadas para mejorar la situación de las poblaciones afectadas en Muisne, Chamanga y Canoa. De esta manera, empleando herramientas etnográficas y georreferenciales, este artículo permite entender la lógica de la aguda verticalidad de la gestión de reconstrucción y los efectos que tiene sobre la población, así como los procesos de reconstitución del tejido socioeconómico.

PalABRAS Clave: Esmeraldas, Manabí, reconstrucción, desastres naturales, derechos humanos, desarrollo humano, indicadores, capacidades, resiliencia.

\section{ABSTRACT}

Based on the framework for human rights indicators for public policy, the present article aims to provide an analysis of the fiscal perspective adopted by the Ecuadorian government as a result of the April 16, 2016 earthquake, focusing particularly on its management of response to overcome this natural disaster. It is argued that this perspective has exacerbated violations to certain human rights, affected resilience-oriented capacities of the affected population, and complicated the establishment of adequate conditions for improving the situation of the affected populations particularly in the coastal areas of Muisne, Chamanga and Canoa. Utilizing

* Docente investigador asociado de la Universidad de las Américas, Sede Ecuador.

** Docente investigador de la Universidad de las Américas, Sede Ecuador.

*** Docente investigador de la Universidad de las Américas, Sede Ecuador.

****Directora de la Carrera de Relaciones Internacionales, Universidad de las Américas, Sede Ecuador. 
both ethnographic and geo-mapping methods, the present article examines the logic behind the distinctly vertical approach to reconstruction and its effects on local populations as well as processes for reforming socio-economic fabrics.

Keywords: Esmeraldas, Manabí, reconstruction, natural disasters, human rights, human development, indicators, capacities, resilience.

\section{INTRODUCCIÓN}

$\mathrm{L}$ a noche del 16 de abril de 2016 Ecuador sufrió un terremoto de 7,8 grados en la escala de Richter, dejando 663 fallecidos, 6.247 personas heridas, 28.775 refugiados en albergues; además de la destrucción de viviendas, edificios e infraestructura (vial, telecomunicaciones, redes de electrificación, servicios básicos) y diversas afectaciones en cantones de diez provincias. ${ }^{1}$

Como resultado de esta catástrofe, se puso en marcha un amplio proceso político, logístico, económico y emocional para la reconstrucción costeña, bajo el recién formado y llamativo Comité de Reconstrucción. La creación de este Comité implicó grandes cambios en la colaboración interinstitucional de política pública y cooperación. A rasgos generales, se hizo rápidamente visible que este proceso tomó una forma y orientación particular, al contrario de algunas experiencias históricas y estándares internacionales recientes, ${ }^{2}$ que abogan para la ampliación tanto de la participación como de la resiliencia a nivel local y de las comunidades con el fin de evitar volver al estado ex ante de la catástrofe, y, por extensión, a un Estado que utiliza información preliminar en vez de resultados concretos para la toma de decisiones.

Según Haque y Etkin, ${ }^{3}$ son las estructuras y procesos del orden anterior los que son con frecuencia responsables de riesgos y vulnerabilidades de la población afectada por el desastre. Por esta razón se estableció a nivel internacional el estándar Building

1. INEC, Reconstruyendo las cifras luego del sismo. Memorias (Quito: Instituto Nacional de Estadística y Censos, 2017).

2. Martín Pallares, "El Comité de Reconstrucción... es el mismo gobierno", Cuatro Pelagatos (blog). 30 de abril de 2016. Disponible en 〈http://4pelagatos.com/2016/04/30/el-comite-de-reconstruccion-es-elmismo-gobierno/>.

3. C. Emdad Haque y David Etkin, edit., Disaster Risk and Vulnerability. Mitigation through Mobilizing Communities and Partnerships (Montreal/Kingston/London/Ithaca: McGill-Queen's University Press, 2012). 
Back Better o $\mathrm{BBB}^{4}$ de la reconstrucción. Sin embargo, los procesos de reconstrucción de característica top-down implican el riesgo de establecer rápidamente el orden y las condiciones "normales" anteriores a la catástrofe que llevó al desastre, más que a un mejoramiento general de las condiciones.

El proceso ecuatoriano de índole acentuadamente top-down en la atención a la emergencia y en la reconstrucción implicó la creación de nuevas instituciones estatales que además se entrecruzaron frecuentemente con las competencias de otras instituciones especializadas (ejemplo: la Secretaría de Gestión Riesgos). Trabajar y planificar desde una aguda verticalidad necesariamente conlleva a perder de vista los derechos, necesidades y aspiraciones de la población al nivel local. Esto se manifiesta particularmente en el trabajo de dos ministerios clave para la atención de la emergencia y la reconstrucción: el Ministerio de Inclusión Económica y Social (MIES) y el Ministerio de Desarrollo Urbano y Vivienda (MIDUVI).

Adicionalmente, desde la perspectiva propuesta del monitoreo de los derechos humanos, resulta altamente problemático el enfoque particular y selectivo que emergió en este proceso, lo cual se deja describir como fusión entre ayuda humanitaria y desarrollo centralizado. Esta fusión particular se aprovechó de la situación pos-sísmica para lanzar una serie de intervenciones estatales con el fin de alterar algunos territorios seleccionados, tejidos económicos-sociales y familiares, relaciones legales en cuanto a la posesión de casas y territorios, así como bases de vida de los damnificados. Esta planificación se diseñó y realizó desde los centros urbanos hacia la periferia costeña con la misma verticalidad mencionada anteriormente, sin tomar en cuenta diferencias sociales, culturales, geográficas y económicas de los "beneficiarios".

Basándonos en una amplia investigación georreferencial y etnográfica, aún en proceso, en las localidades de Chamanga, Muisne (Esmeraldas), así como Canoa y Jama (Manabí), este artículo tiene el doble propósito de analizar, en un primer paso, la ceguera de la planificación estatal top-down hacia las poblaciones y comunidades locales y sus condiciones de vida. Esta ceguera implica el peligro de capitalizarse política y económicamente sobre las múltiples vulnerabilidades de las personas afectadas.

En segundo lugar, al indicar estos efectos en términos de posibles violaciones de derechos humanos, se plantea entonces un debate amplio sobre las diferentes perspectivas legales y políticas con las que se puede abordar analíticamente lo que caracterizamos como una nueva fusión emergente entre las respuestas de las instituciones

4. GFDRR et al., "Guide to Developing Disaster Recovery Frameworks. Sendai Conference Version", en Disaster Recovery Framework (Washington D. C.: Global Facility for Disaster Reduction an Recovery/ Banco Mundial/PNUD/Unión Europea, 2015), 11-2. 
públicas, la ayuda humanitaria y las intervenciones "desarrollistas", en el contexto de la reconstrucción.

Con este objetivo, analizamos este proceso desde dos perspectivas: 1) desde los indicadores de derechos humanos (DD. HH. en adelante) y su uso por el gobierno ecuatoriano entre 2009-2014; y 2) desde la mirada del desarrollo humano, ${ }^{5}$ es decir, del enfoque de capacidades y su acentuación metodológica del individuo para la construcción de las políticas públicas. Esta segunda perspectiva se justifica, por un lado, por su integración en la Constitución vigente de 2008; y, por otro, porque el enfoque del monitoreo de DD. HH. se basa en cierta medida en la epistemología subyacente al desarrollo humano.

\section{SIDERECHOS Y EL DESARROLLO HUMANO}

En este apartado, particularmente teórico, introducimos el enfoque de los indicadores de derechos humanos en el marco de la teoría del desarrollo humano, con el fin de analizar nuestros resultados del trabajo de campo sobre la reconstrucción. En la última ronda del Examen Periódico Universal (EPU) de las Naciones Unidas en Ginebra en mayo de 2017, el equipo de delegados ecuatorianos se defendió frente a preguntas y cuestionamientos críticos de veintiocho países sobre la protección y la realización de derechos humanos en el país, destacando la exitosa creación de un sistema integral de información sobre DD. HH., llamado SIDERECHOS. ${ }^{6}$

Este sistema de información es el producto preliminar de un esfuerzo de carácter internacional para crear indicadores cuantitativos y cualitativos con el fin de medir todas las políticas públicas de un país a la luz de los DD. HH.. Anteriormente, Ecuador fue reconocido como el primer país del mundo en trabajar en la realización de un sistema integral, que tenía como particularidad la estructuración u orientación del sistema internacional, adaptado a las necesidades domésticas. Sin embargo, este proceso culminó en el año 2014 sin mayor éxito. ${ }^{7}$ Además, el resultado de este proceso largo

5. Entre otros, Amartya Sen, Development as Freedom (Oxford/New York: Oxford University Press, 1999); Martha Nussbaum, Frontiers of Justice; Disability, Nationality, Species Membership (Cambridge: Harvard University Press, 2006); Martha Nussbaum, Creating Capabilities: The Human Development Approach (Cambridge: Belknap Press of Harvard University Press, 2011); Sabina Alkire, "Human Development: Definitions, Critiques, and Related Concepts", OPHI Working Papers, No. 36 (2010). Disponible en 〈http://www.ophi.org.uk/human-development-definitions-critiques-and-related-concepts〉.

6. Consejo de Derechos Humanos, Informe nacional presentado con arreglo al párrafo 5 del anexo de la resolución 16/21 del Consejo de Derechos Humanos-Ecuador (Ginebra, 4 de julio de 2017), 5, 21, 22.

7. Johannes M. Waldmüller, "Human Rights Indicators as 'Development 2.0'?”, Alternautas, No. 1 (2014): 76-87; Johannes M. Waldmüller, “ 'Living Well Rather Than Living Better’ Measuring Biocentric Human- 
e intenso por parte de instituciones internacionales y nacionales no es accesible de manera pública, por lo que si existiera y fuera público actualmente, sería solamente de forma debilitada y rudimentaria, en comparación con el diseño complejo disponible, por ejemplo, para algunos estados federales de México. ${ }^{8}$

El proceso de SIDERECHOS en Ecuador inició bajo el mandato del Ministerio de Justicia, Derechos Humanos y Cultos, en el año 2009, después de la visita del experto de la Oficina del Alto Comisionado para los Derechos Humanos (ACNUDH) al país. Siguiendo su diseño, ${ }^{9}$ la oficina con sede en Quito elaboró, con el apoyo de la oficina mexicana experta en la temática y varias otras instituciones en el país, un estudio piloto para una serie de derechos seleccionados con el fin de ampliar las bases de datos en los siguientes años. La idea principal fue implementar y nutrir una plataforma interactiva que retomaría la división de indiciadores propuestos por la ACNUDH a nivel internacional. De esta manera, se procedió a subdividir cada "derecho humano [...] a tres tipos de indicadores: estructurales (bases legales), de proceso (metas de políticas públicas) y de resultado (evaluando los objetivos plasmados y logros reales).

En consecuencia, se buscó implementar un eje transversal relevante para este estudio: la desagregación de datos para cada indicador a los niveles más reducidos posibles (por ejemplo, según género, religión, etnicidad, edad, rural, urbano, etc.). El objetivo detrás de SIDERECHOS fue cerrar la brecha de "aplicabilidad" entre métodos y generación de conocimiento en el campo compartido de desarrollo, ayuda humanitaria y DD. HH. ${ }^{10}$ En otras palabras, se trató de aumentar la operatividad de los DD. HH. en vista de iniciativas medibles como los Objetivos del Desarrollo Sostenible; además de hacer a los derechos mismos, más visibles y tangibles. Adicionalmente, la esperanza fue que, a través de los indicadores de los DD. HH., la sociedad civil sería empoderada para demandar que se respeten y garanticen cada vez más estos derechos, como resultado de que, mediante información constante, coherente y desagregada, se facilite

Nature Rights and Human-Nature Development in Ecuador", Journal of Social Quality, No. 2 (2015): 7-28. Disponible en 〈https://doi.org/10.3167/IJSQ.2015.050202〉.

8. UN ACNUDH, Construcción de Indicadores de Derechos Humanos: Experiencias Regionales (México: UNHCHR, 2013); UN ACNUDH, Informe de misión. Asistencia técnica al proyecto SIDERECHOS, Septiembre 2010 (Quito, 2010); UN OHCHR, Human Rights Indicators: A Guide to Measurement and Implementation (Nueva York/Ginebra: UN OHCHR, 2012).

9. UN OHCHR, "Report on Indicators for Promoting and Monitoring the Implementation of Human Rights", International Human Rights Instruments (Ginebra: UN OHCHR, 2008). Disponible en 〈http://www2. ohchr.org/english/bodies/icm-mc/docs/HRI.MC.2008.3EN.pdf). UN OHCHR, Human Rights Indicators: A Guide to Measurement and Implementation.

10. Gauthier de Béco, "Human rights indicators and MDG indicators: building a common language for human rights and development organizations", en Paul Gready y Wouter Vandenhole, edit., Human Rights and Development in the New Millenium (Oxon/New York: Routledge, 2014), 50-70. 
el monitoreo civil, para así fortalecer la transparencia en cuanto a la implementación de las políticas públicas.

Actualmente la falta de disponibilidad de SIDERECHOS revela una incoherencia importante frente a la lógica inicial del diseño, la promoción y la protección de DD. HH. en el país. Sin embargo, esta perspectiva particular de los indicadores de DD. $\mathrm{HH}$. sirve como marco metodológico y analítico para evaluar los resultados preliminares de la investigación.

En los casos particulares de Esmeraldas y Manabí, se presenta una falta o carencia de consideraciones, como son las realidades individuales y locales, es decir, del nivel "desagregado" de conocimiento, que de hecho sería necesario para responder y ampliar adecuadamente la protección y realización de los DD. HH. con respecto a la población particularmente vulnerable después del terremoto.

Íntimamente vinculado con la metodología de indicadores sobre DD. HH. se encuentra también otro enfoque relevante para la discusión presentada: el desarrollo humano, ${ }^{11}$ concepto que se incluyó también en la Constitución de $2008,{ }^{12}$ así como en los Planes Nacionales del Buen Vivir (2009-2013 ${ }^{13}$ y 2013-2017 $\left.{ }^{14}\right)$. El mismo término también sirvió para nombrar el programa nacional de transferencias condicionales "Bono del Desarrollo Humano", que, como analizaremos, se convirtió en pieza clave en términos de control social y financiero para la posterior entrega de casas reconstruidas mediante copago.

Según varias fuentes, la idea inicial de hacer los DD. HH. "tangibles" y aptos al monitoreo a través de indicadores surgió en vista del éxito de las indicadores del desarrollo humano elaborados por Amartya Sen, filósofo y premio Nobel en Economía de 1998, y Mahbub Ul-Haq, durante los años de 1990 para el Programa de las Naciones Unidas para el Desarrollo (PNUD). ${ }^{15}$

11. Nussbaum, Frontiers of Justice; Disability, Nationality, Species Membership; Sen, Development as Freedom; Alkire, "Human Development: Definitions, Critiques, and Related Concepts"; Nussbaum, Creating Capabilities: The Human Development Approach; Séverine Deneulin y Lila Shahani, edit., An Introduction to the Human Development and Capability Approach: Freedom and Agency (London: Earthscan, 2010).

12. Ecuador, Constitución Política de la República del Ecuador (2008), art. 59.

13. Secretaría Nacional de Planificación y Desarrollo (SENPLADES), Plan Nacional de Desarrollo. Plan Nacional para el Buen Vivir 2009-2013. Construyendo un Estado plurinacional e intercultural (Versión resumida) (Quito: SENPLADES, 2009), 28, 31, 32, 38, 49, 53, 72, 74, 258.

14. SENPLADES, "Buen Vivir Plan Nacional 2013-2017" (Quito: SENPLADES, 2013), 28, 66, 12, 222.

15. Eibe Riedel, Gilles Giacca y Christophe Golay, "The Development of Economic, Social, and Cultural Rights in International Law: Introduction", en Eibe Riedel, Giacca Gilles y Christophe Golay, edit., Economic, Social, and Cultural Rights in International Law (Oxford: Oxford University Press, 2014), 3-48; 
El postulado principal del desarrollo humano es tomar en consideración lo que los individuos valoran como base para la construcción de políticas públicas, incluso las de gestión de riesgos. En otras palabras, el desarrollo humano se centra en "lo que hace que nuestra vida humana tenga valor". ${ }^{16}$ Esto quiere decir que, en lugar de construir políticas desde arriba hacia abajo, se debería primero sondear lo que se valora y es importante para las personas al nivel local, así como sus capacidades y posibilidades. ${ }^{17}$ En esta visión son los individuos quienes deben ser los sujetos del desarrollo, son ellos quienes sientan medidas y fines del desarrollo. ${ }^{18}$

La evaluación del desarrollo debe ser realizada estimando "si las libertades de los individuos han sido ampliadas", ya que el desarrollo debería lograrse por medio de la libre agencia de los individuos". ${ }^{19}$ Así, Sen afirma que en las actividades del desarro1lo, "los individuos han de verse como seres que participan activamente-si se les da la oportunidad- en la configuración de su propio destino, no como meros receptores pasivos de los frutos de ingeniosos programas de desarrollo". ${ }^{20}$ Desde esta perspectiva, el desarrollo se basa en las libertades de las personas, entendidas como oportunidades que estas tienen para tomar decisiones y avanzar en los objetivos principales de sus vidas. De manera que los individuos deben decidir qué tipo de desarrollo desearían para sí mismos. Además, es fundamental que estas oportunidades requieran un marco legal-político adecuado. De ahí la conexión entre el monitoreo de los DD. HH. en las políticas públicas y el enfoque del desarrollo humano. Solo cuando los individuos y los grupos sociales se reconocen como agentes, pueden definir sus prioridades y elegir los mejores medios para alcanzarlas.

El gobierno del Ecuador en los últimos años ha puesto un mayor énfasis en las oportunidades de participación e inclusión del individuo dentro de la sociedad. Ha buscado implementar el concepto de Buen Vivir como enfoque de desarrollo humano en el país, resaltando la importancia de las relaciones participativas del ser humano

De Béco, "Human rights indicators and MDG indicators: building a common language for human rights and development organizations".

16. Sen, Development as Freedom.

17. Basándose en un estudio profundizado de los aportes culturales y filosóficos de la India y del Occidente, la filósofa Martha Nussbaum presentó una lista de las diez capacidades fundamentales. Nussbaum, Frontiers of Justice; Disability, Nationality, Species Membership, considerando elementos como la soberanía corporal, la posibilidad de jugar y soñar, algunos necesidades básicas económicas y materiales así como libertades políticas y del pensamiento.

18. Séverine Deneulin, "Beyond Individual Freedom and Agency: Structures of Living together in the Capability Approach", en Flavio Comim, Mozaffar Qizilbash y Sabina Alkire, edit., The Capability Approach: Concepts, Measures and Applications (Cambridge: Cambridge University Press, 2008), 107.

19. Sen, Development as Freedom, 4.

20. Ibíd., 75 . 
con su familia, comunidad local, gobierno nacional y medioambiente en particular. ${ }^{21}$ Dentro de esta política, las nociones de libertad del individuo y autonomía de los pueblos, junto con la coexistencia e inclusión social, mencionados por Catherine Walsh, ${ }^{22}$ no necesariamente se contraponen, sino que trabajan en conjunto para crear una sociedad que -en teoría - no solo tiene la posibilidad de participar activamente, sino que es capaz de participar efectivamente en varios niveles del Estado. Esto está reflejado en el Objetivo 8 del "Plan Nacional Para el Buen Vivir 2013-2017", que trata de "fortalecer las capacidades y potencialidades de la ciudadanía". ${ }^{23}$

Para el siguiente análisis, este marco combinado entre el enfoque de capacidades e indicadores de DD. HH. es altamente relevante, ya que las políticas públicas del Ecuador deben ser guiadas por los mismos enfoques, tanto a nivel de gestión de riesgos ex ante, ${ }^{24}$ como de reconstrucción ex post. Ambos marcos teóricos-políticos subrayan como algo imprescindible el enfoque a lo individual, así como adaptar las intervenciones estatales y privadas a las aspiraciones, capacidades y derechos de cada persona afectada según sus propias características y necesidades.

Entonces, lo que sigue es una descripción de los casos analizados aquí: el desalojo forzado en Muisne y Chamanga, en Esmeraldas, justificado por un estudio de riesgo elaborado por la Escuela Politécnica del Ejército y la Dirección Nacional de Defensa Civil ${ }^{25}$ hace varios años, y sin sustento por otros estudios corroborativos. Estos casos son puntualmente completados por algunas observaciones sobre la situación de la reconstrucción en Canoa, Manabí.

La metodología empleada para la recolección de datos comprende estancias repetidas en el campo (julio y diciembre 2016, enero y abril 2017), la realización de veinte entrevistas semiestructuradas en las tres comunidades, y en Quito, además de un sinnúmero de conversaciones informales y grabaciones de fotos y videos aéreos en diferentes lugares.

21. Ryan J. Cobey y M. L. Bandeira, "Sumak Kawsay or Buen Vivir, an alternative model in the Andean State?", Farol, Revista de Estudos Organizacionais e Sociedade, No. 3 (2010): 481-537.

22. Catherine Walsh, "The (Re)articulation of Political Subjectivities and Colonial Difference in Ecuador: Reflections on Capitalism and the Geopolitics of Knowledge", Neplanta: Views from South, No. 1 (2002).

23. SENPLADES, "Buen Vivir Plan Nacional 2013-2017”.

24. Abhas K. Jha et al., Safer Homes, Stronger Communities: A Handbook for Reconstruction after Natural Disasters (Washington D. C.: The International Bank for Reconstruction and Development/The World Bank, 2010).

25. Mario A. Cruz DeHowitt, María Cristina Acosta y Nelson Eddy Vásquez, Riesgos por tsunami en la Costa ecuatoriana (Quito: Escuela Politécnica del Ejército, s. f.). 


\section{BIOPOLÍTICA Y COACCIÓN EN LA ADMINISTRACIÓN DE DESASTRES}

El terremoto de abril de 2016 repercutió particularmente en las provincias de Esmeraldas y Manabí, donde hubo diversos grados de afectaciones. Ante esta eventualidad, el gobierno desplegó funcionarios de múltiples instituciones (MIES, MIDUVI, Instituto Nacional de Estadística y Censos/INEC, Comando Conjunto, Policía, Bomberos, entre otras) para atender a la población, levantar información de estimación de daños en diferentes áreas y posteriormente para restaurar ciertos niveles de "normalidad".

Según las directrices internacionales, dentro de las primeras setenta y dos horas se debe levantar una Evaluación de Daños y Análisis de Necesidades (EDAN). Sin embargo, el uso de esta herramienta fue suspendida en el año 2014, y fue reemplazada por una Evaluación Inicial de Necesidades (EVIN) que fue diseñada con apoyo del Programa Mundial de Alimentos (PMA). En el caso del terremoto, dicha evaluación se realizó el 20 de abril de $2016,{ }^{26}$ lo que demuestra que las decisiones se tomaron de acuerdo con los planes de contingencia pensados para la posible emergencia de erupción del volcán Cotopaxi en 2015, considerando que el gobierno estableció alerta naranja y se inició un amplio proceso de prevención a nivel nacional.

Por la magnitud del fenómeno, tal cual establece la Estrategia Internacional de Reducción de Desastres, ${ }^{27}$ el gobierno realizó la Declaratoria de Estado de Excepción (en las provincias de Esmeraldas, Manabí, Santa Elena, Santo Domingo, Los Ríos y Guayas $)^{28}$ para disponer de los recursos jurídicos, económicos y políticos para intervenir en las zonas de desastres, y en el resto del país para asegurar ciertos niveles de gobernabilidad. Posteriormente, el gobierno decretaría un Estado de Excepción para las provincias de Esmeraldas y Manabí con el fin de asegurar y "coordinar esfuerzos a fin de ejecutar las acciones necesarias indispensables para mitigar y prevenir los riesgos". ${ }^{29}$

Este tipo de intervenciones buscaron reinstaurar la "normalidad" mediante una pronta provisión de bienes y servicios para la población afectada. En el caso ecuatoriano identificamos en las actuaciones gubernamentales el predominio de una perspectiva fisicalista, que concibe los desastres como eventos físico-naturales externos a

26. Información disponible en Plan V, La SGR, de espaldas al riesgo (Quito, 2016). htttp://www.planv.com. ec/investigacion/investigacion/la-sgr-espaldas-al-riesgo〉. Consulta: 12 de diciembre de 2017.

27. S. Bass, R. Selvaraju, Dey de Pryck y F. Battista, Análisis de sistemas de gestión del riesgo de desastres (s. 1.: Organización de las Naciones Unidas, 2009).

28. Decreto Presidencial No. 1001, firmado el 17 de abril de 2016.

29. Decreto Presidencial No. 1215, firmado el 14 de octubre de 2016. 
las voluntades humanas, imprevisibles y por tanto incontrolables que afectan la vida social. ${ }^{30}$ No obstante, lo más relevante es que esta visión tiende a cuantificar los efectos (fallecidos, edificios destruidos, infraestructura afectada, etc.) para implementar medidas y reconstruir la infraestructura y aparato productivo. Según Lavell, este tipo de perspectiva y manejo subordina las dinámicas sociales al desastre, que de otro modo habrían sido "interrumpidas" por el terremoto, y la solución pasaría por reinstaurar un "orden", reconstruir las instalaciones y los edificios para que "la población vuelva a su vida normal". ${ }^{31}$

Los estudios de desastres más recientes han demostrado que son las condiciones socioeconómicas vulnerables y precarias previas a la catástrofe las que se convierten en un verdadero desastre humano. ${ }^{32}$ En lugar de volver al estatus anterior, lo cual significaría ningún mejoramiento cualitativo, se ha sugerido a través de estándares internacionales buscar y ampliar la resiliencia local por medio de un proceso de desarrollo sostenible local a largo plazo. ${ }^{33}$ Para alcanzarlo es fundamental construir las políticas públicas de atención a partir de las voces de los actores locales, lo que además plantea el marco legal-político de SIDERECHOS.

Otro documento que deber ser considerado es la Carta Humanitaria impulsada por el Proyecto Esfera ${ }^{34}$ que establece tres derechos fundamentales: derecho a vivir con dignidad, derecho a recibir asistencia humanitaria, derecho a la protección y seguri$d a d$, que consideran cuatro principios de protección:

1. Evitar exponer a las personas a daños adicionales como resultado de las acciones;

2. Velar para que las personas tengan acceso a una asistencia imparcial;

3. Proteger a las personas de los daños físicos y psíquicos causados por la violencia y coerción;

30. Allan Lavell Thomas, "Ciencias sociales y desastres naturales en América Latina: un encuentro inconcluso", Revista Mexicana de Sociología, No. 2 (abril-junio 1993): 253-85.

31. Ibíd.

32. Haque y Etkin, edit., Disaster Risk and Vulnerability. Mitigation through Mobilizing Communities and Partnerships; Ann-Margaret Esnard y Alka Sapat, Displaced by Disaster. Recovery and Resilience in a Globalizing World (Nueva York/Oxon: Routledge, Taylor \& Francis, 2014).

33. Overseas Development Institute, The future framework for disaster risk reduction. A guide for decisionmakers (Londres: ODI, 2014); Abhas K. Jha et al., Safer Homes, Stronger Communities: A Handbook for Reconstruction after Natural Disasters.

34. El Proyecto Esfera fue iniciado en 1997 por un grupo de organizaciones no gubernamentales y el Movimiento Internacional de la Cruz Roja y de la Media Luna Roja, a fin de elaborar un conjunto de normas mínimas universales en ámbitos esenciales de las respuestas humanitarias. 
4. Ayudar a las personas a reivindicar sus derechos, reparación y recuperarse de los efectos de los abusos sufridos. ${ }^{35}$

Sin embargo, muchos de estos principios no fueron acatados; en cambio, las acciones promovidas por el gobierno vulneraron los derechos y sus consiguientes principios, tal como más adelante lo expondremos.

El manejo fisicalista de desastres tiende a vulnerar algunos derechos humanos de las personas ya afectadas y vulnerables. Una de las medidas que refuerzan esta inadvertencia y la de efectos psicológicos y afectivos de la población, por ejemplo, es la Declaratoria de Emergencia, que de alguna manera establece "el tiempo que durará el desastre" y, así, la posibilidad de suspensión de normas legales, libertades y derechos, con el justificativo de necesitar recursos jurídicos, económicos y logísticos para atender el desastre y tener márgenes de gobernabilidad en todo el territorio. Así, la declaratoria se convierte en una especie de "estrategia combinada de cooptación-represión y de prácticas clientelares y corporativas", ${ }^{36}$ dejando al margen la participación de la sociedad civil.

El estado de excepción habilita al gobierno para realizar acciones que justifiquen vulneración de derechos humanos, pues el decreto suspende, por ejemplo, el derecho a la vivienda (estipulado además en detalle en el diseño de SIDERECHOS), y permite la requisición de personas en tránsito. Según el derecho a vivir con dignidad, el espacio en un albergue debe ser de 3,5 metros por persona, sin incluir otras áreas (cocina, baños, comedores, almacén), derecho que no se cumplió, pues las carpas tenían cerca de doce metros cuadrados para una familia, en las que habían más de cuatro integrantes.

Además, el estado de excepción como herramienta jurídica es atentatoria frente a los desbordes sociales que exigen mayor atención o provisión de alimentos, medicamentos u otros suplementos necesarios de subsistencia. El régimen de emergencia establece el desplazamiento de las poblaciones, sobre todo para asegurar la situación y poder establecer zonas de control, como los así llamados "albergues". Estos campamentos $^{37}$ son estacados vigilados por cámaras que "constituyen espacios de gestión de

35. Manual de Esfera, Carta Humanitaria y normas mínimas para la respuesta humanitaria (s. 1.: Proyecto Esfera, 2011), 139.

36. Daniel Rodríguez Velásquez, "La política pública frente a desastres en el contexto de la reforma del Estado: opciones desde la sociedad civil”, en Políticas públicas y desastres (México D. F.: Cooperación Internacional, 2008), 44.

37. Se establecieron tres en Muisne, uno en Canoa y uno en Chamanga, respectivamente. 
la vida: confinan sufrimientos sociales vueltos invisibles, formas de violencia que no son computadas por las estadísticas, y se convierten en espacios de abandono social". ${ }^{38}$

Este fue el caso de los albergues en Muisne, en donde la población vivía bajo un régimen militarizado, con horarios estrictos para cocinar, bañarse, despertar, dormir, recibir visitas y otros aspectos centrales de la vida cotidiana; si bien en las directrices internacionales esto es necesario para establecer cierto orden en los recintos, lo que va en contra es el tiempo de duración de los albergues, pues la Carta Humanitaria establece un tiempo reducido para su duración (entre dos y cinco días), pero en este caso los recintos duraron alrededor un año y quince meses. Durante ese período nadie tenía formalmente permiso de trabajar, lo que no solamente choca con algunos derechos fundamentales, sino que además parecía absurdo frente a la situación precaria de los damnificados.

Así, en adelante esta realidad se constata con el testimonio de una habitante del albergue 1, con quien logramos conversar fuera del campo; a pesar de la prohibición de entrada para cualquier persona no gubernamental:

Al principio, cuando llegaron donaciones de todo lado del país, del exterior, sí recibíamos
todo, pero luego el MIES y el ejército acumulaban las donaciones en los centros de acopio,
y todo lo llevaban a las carpas militares y desaparecía. Con el paso del tiempo recibimos
cada vez menos, ni agua tenemos, cuando alguien trae un poco, el resto le pide, porque esas
carpas son calientes y ahí todo el mundo se sofoca. Y tampoco podemos recibir ayuda o do-
nación, porque los del MIES o militares se atajan y no dejan que nos den [...]. No podemos
sacar fotos, no hay internet, no podemos recibir visitas; si viene la prensa o gente de afuera,
estamos prohibidos de quejarnos, nos amenazan con sacarnos del albergue y no recibir las
casas que nos prometieron o la ayuda; por eso callamos. ${ }^{39}$

Este régimen de censura en los albergues fue posible gracias a un instrumento elaborado y creado para el control de la población, nos referimos al Registro Único de Damnificados (RUD), que es la base de datos elaborada por el INEC en coordinación con otras instancias gubernamentales. Esta herramienta se supone que contiene datos personales, estatus de damnificación, ubicación geográfica y el tipo de política de la que será beneficiado (casa, crédito, bono u otro):

Ese registro se alimentó de toda la riqueza de la base de datos de albergues y refugios, podíamos identificar el tipo de damnificado, si había perdido su casa, si había perdido un

38. Víctor Marchezini, "La producción silenciada de los desastres naturales en catástrofes sociales", Revista Mexicana de Sociología, No. 2 (2014): 263.

39. Entrevisa 1: damnificada viviendo en el albergue No. 1 de Muisne. Anónima por protección a la fuente, Muisne, 2 de abril de 2017. 
miembro de familia, etc. [...]. El Registro Único de Damnificados nos interesaba identificar a la persona, llegar a ella con preguntas mucho más específicas y mucho más rápidas. Ahora, el Registro Único de Damnificados se fue constituyendo en una base grande. [...] entonces se definió el concepto damnificado en base a tres características: la persona que había perdido su vivienda; la persona que había perdido un miembro del hogar, que había muerto; o aquella que había perdido sus medios de vida. ${ }^{40}$

Sí bien fue creado para tener una base de datos de la población, y de esa forma tener una vinculación con la sociedad civil y articularse para la mitigación y atención, el RUD se constituyó en una herramienta que refuerza la lógica top-down ejecutada por el gobierno, pues a diferencia del EDAN no tenía como objetivo analizar daños sino verificar afectados. Una prueba de ello fue la exclusión de un amplio número de damnificados, debido a que no todos aceptaron vivir en los albergues, ya sea por las condiciones de los mismos (excesivo control, escasa privacidad, restricción para retomar sus vidas) o porque decidieron volver a sus hogares para cuidar los restantes bienes antes de ser robados o saqueados.

Lo anterior atenta contra los principios de protección de la Carta Humanitaria, ya que las personas no recibieron asistencia imparcial sino condicionada, no se evitó daños a causa de las acciones gubernamentales, se ejerció coerción sobre los afectados, y no se ayudó a reivindicar otros derechos humanos. Según las evidencias del campo, muchas personas en Esmeraldas y Manabí resultaron marginadas de la distribución de algunos bienes (kits alimenticios, carpas y otros), es decir, no recibieron atención del gobierno, pues un requisito para ser beneficiario era quedarse en los albergues y someterse al régimen de control, lo que demostraba la ineficiencia del RUD. Además, como indican los siguientes testimonios, hubo casos de personas no damnificadas que recibieron ayuda. Esta práctica pudimos documentarla en varios otros ámbitos, en particular, en la entrega de casas por parte del MIDUVI en las zonas rurales de Canoa y Jama:

Hemos sido encuestados por todo el mundo, dos, tres veces, pero aquí nos ve olvidados. Las donaciones son para la gente de los campamentos, al principio recibimos algunas cosas, sobre todo alimentos, y por ello damos gracias, pero luego nos marginaron. Por suerte vinimos a este terreno de un familiar y como sea logramos construir este solar... No todos los afectados recibimos cosas, es más, algunos que no necesitaban y que tampoco fueron damnificados, recibieron incluso casa; las razones no las sabemos. ${ }^{41}$

40. INEC, Reconstruyendo las cifras luego del sismo. Memorias, 32.

41. Entrevista 2: damnificada, anónima por protección a la fuente, Chamanga, 3 de abril de 2017. 
Los problemas de la identificación y distribución correcta de las casas igualmente han sido señalados por parte del ejecutivo. Queda, sin embargo, el interrogante sobre la efectividad y por tanto el propósito verdadero del registro detallado del INEC:

La distribución de las casas es un tema muy complicado, a pesar de que el gobierno trata de hacerlo mejor siempre aparece un vivo, por eso siempre pedimos que la población nos acompañe, pues a veces reciben la casa pero luego los pobladores dicen que "ese no era el dueño", ya no es culpa del gobierno, la gente debe decir eso, para evitar cometer errores, porque la gente no avisa, les decimos que hablen, pero a veces no lo hacen por no enemistarse con el vecino o cosas así...

El control poblacional ejercido por el gobierno, de alguna manera se parece a lo que Foucault analizó como biopolítica, es decir "incidir sobre la vida, administrarla, organizarla, regularla e inhibirla; se amplifica el rol de la misma en la existencia humana". ${ }^{42}$ Esto implica su estudio o generación de información a través de censos, encuestas u otros instrumentos científicos que permitan ese fin. La biopolítica ejercida por el gobierno no solo discrimina entre los que viven y los que decidieron no vivir en los albergues, sino que también condiciona a ambos para recibir ayuda; de esa forma, incide sobre sus hábitos y rutinas para tener control de los posibles reclamos y peticiones de la sociedad civil. Este tipo de régimen atenta contra el derecho de vivienda digna, pues el otorgamiento de las casas está condicionada, y los campamentos estatales, por su carácter temporal, son precarios en condiciones de seguridad, privacidad y servicios básicos.

Otro ejemplo de esta intervención centralizada se refiere a los tejidos sociales y familiares. Bajo pretexto de "aprovecharse de la reconstrucción para poner orden", en palabras del MIDUVI Esmeraldas, se iban a modificar los núcleos familiares (extendidos en la Costa) bajo un mismo techo. Las casas otorgadas por el MIDUVI no permiten más de cuatro familias bajo un techo con los espacios reducidos (cuarenta metros cuadrados), con el fin -por lo general- de limitar "pasivamente" el crecimiento de familias vistas como pobres y precarias. Adicionalmente, sobre todo aquellas de las urbanizaciones, tuvieron el carácter de permanente, aunque muchas no cumplen con los parámetros de la Carta ya sea en superficie o materiales (algunas de plancha de metal, otras de madera), y tampoco aseguran próximas condiciones dignas, pues están en zonas de riesgo (inundación, tsunami u otros); tal es el caso de la zona de Bunche, que, a pesar de que es la nueva urbanización para los afectados de Muisne, también podría verse afectada por un tsunami. En caso de un desastre, la situación de Bunche

42. Michel Foucault, Nacimiento de la biopolítica. Curso del Collège de France (1978-1979) (Madrid: Akal, 2009), 75 . 
se agrava por la existencia de una única ruta de escape y completamente rodeada por camaroneras.

Según los interlocutores entrevistados, no había ninguna encuesta referente a las construcciones o instalaciones que indaguen sobre las necesidades y aspiraciones de la gente afectada. En este sentido, el gobierno a través de diferentes maneras presionó a la gente a someterse, atentando de esa forma múltiples derechos y reduciendo posibilidades de mejorar sus condiciones, todo ello respaldado con el argumento que "el gobierno quiere su seguridad":

Lo que sí le falta a Chamanga es ordenamiento porque es una parroquia que ha crecido en un total desorden, no habido un lineamiento de calles, nada, se ha puesto en un relajo, no habido un control, necesitamos que el Municipio junto con la Junta Parroquial realice un verdadero ordenamiento, para que se vea bonito y así vaya cambiando. ${ }^{43}$

\section{VERTICALIDAD Y CEGUERA LOCAL}

Estas estructuras de verticalidad son igualmente reproducidas a nivel cantonal. Si bien el gobierno nacional busca que los municipios trabajen en conjunto con los GAD parroquiales para asegurar un mayor ordenamiento de las parroquias afectadas por el terremoto, el poder de decisión aún se queda dentro del propio municipio en algunos casos.

Durante una entrevista con el presidente del GAD Parroquial de Canoa, y otros representantes actuales, se mencionó que la participación del pueblo de Canoa en el uso de los suelos es muy escasa. Según ellos, los proyectos de reconstrucción realizados en Canoa son notificados a las autoridades locales, pero la toma de decisiones se queda específicamente dentro de la competencia del Municipio sin contar con la participación del GAD Parroquial. ${ }^{44}$

En particular, el Municipio de San Vicente ha realizado varios proyectos propios de reconstrucción en la región, que incluye la construcción de un conjunto de diez casas nuevas en Canoa utilizando recursos de cooperación ofrecidos por la empresa internacional DIRECTV.

Cerca de la playa en Canoa se encuentran varios espacios vacíos donde existían hoteles, hostales y restaurantes antes del terremoto. Según el GAD Parroquial, el Mu-

43. Entrevista 3: directora provincial del MIDUVI, Esmeraldas, 3 de abril de 2017.

44. Entrevista 4: presidente del GAD Parroquial de Canoa, 2 de junio de 2017. 


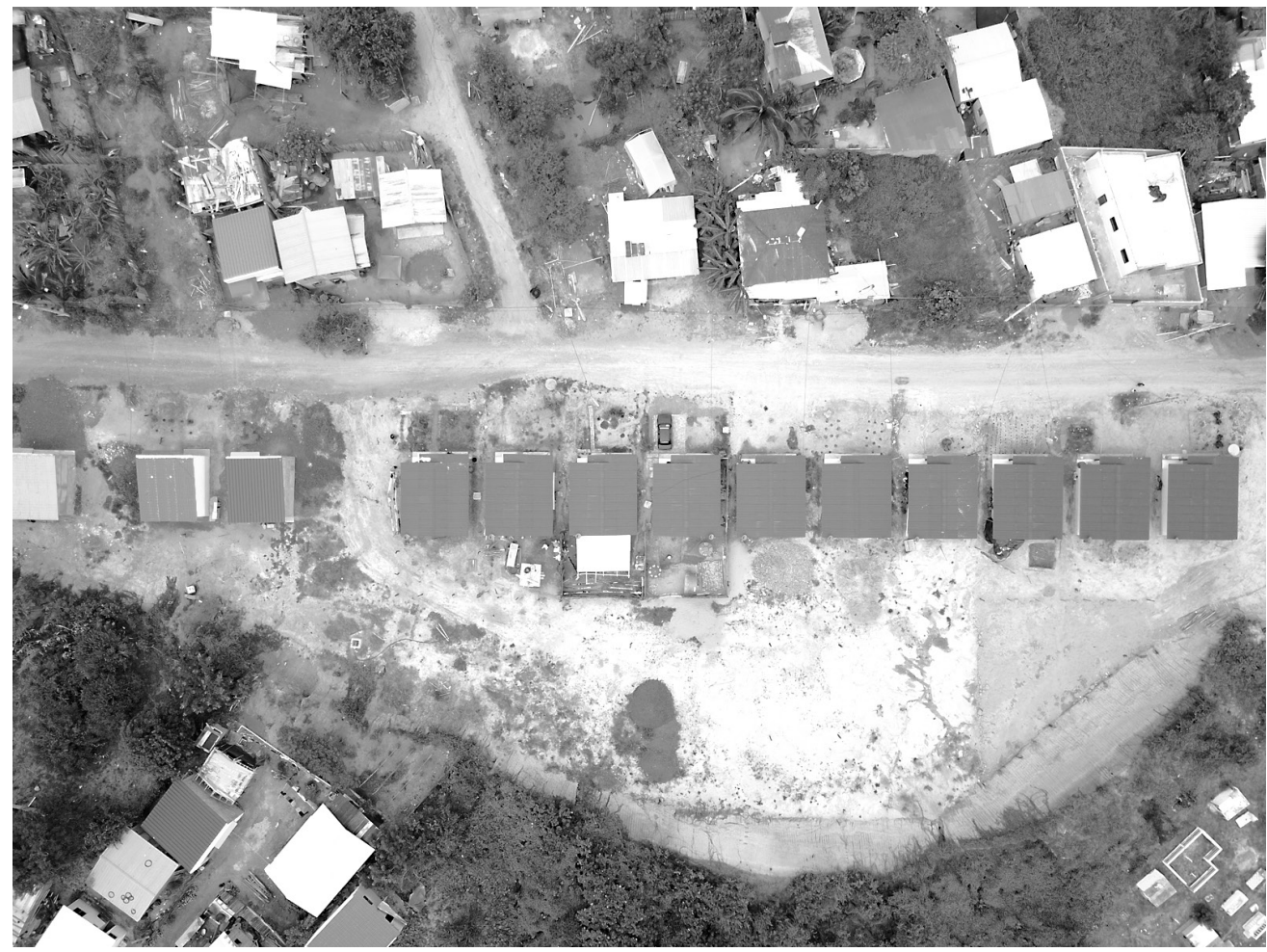

Figura 1: Vista aérea del conjunto de casas construidas a través del proyecto del Municipio de San Vicente con la empresa DIRECTV. Hay dos viviendas ubicadas a la izquierda del conjunto, construidas por el MIDUVI. Se pueden observar otras viviendas similares alrededor. Fotografía propia.

nicipio de San Vicente se encargó de la planificación para el uso del suelo, reflejado en la figura 2, donde fue el sitio de un hotel construido con capital extranjero.

Hoy se tiene previsto reubicar todos los restaurantes y bares que están actualmente en la playa para así crear espacio para dos nuevos complejos turísticos con inversión extranjera. Aunque el Municipio de San Vicente tiene el poder de decisión en este caso, los planes de reconstrucción de este suelo no cuentan con la participación del GAD Parroquial, y tampoco con la población afectada. En efecto, es probable que un cambio de este tipo afectaría profundamente los tejidos socioeconómicos entre pescaderos artesanales y servicios gastronómicos.

Otro aspecto sin tomar en cuenta sobre las necesidades locales es la distribución de casas. Así, para obtener una casa del MIDUVI, la entrega fue condicionada según el mismo patrón (en Manabí y Esmeraldas), ya sea que las personas estén dentro los albergues o para los que no quisieron vivir en ellos. Como resultado, los damnificados debían cumplir con ciertos requisitos para recibir la tan ansiada ayuda, aunque 


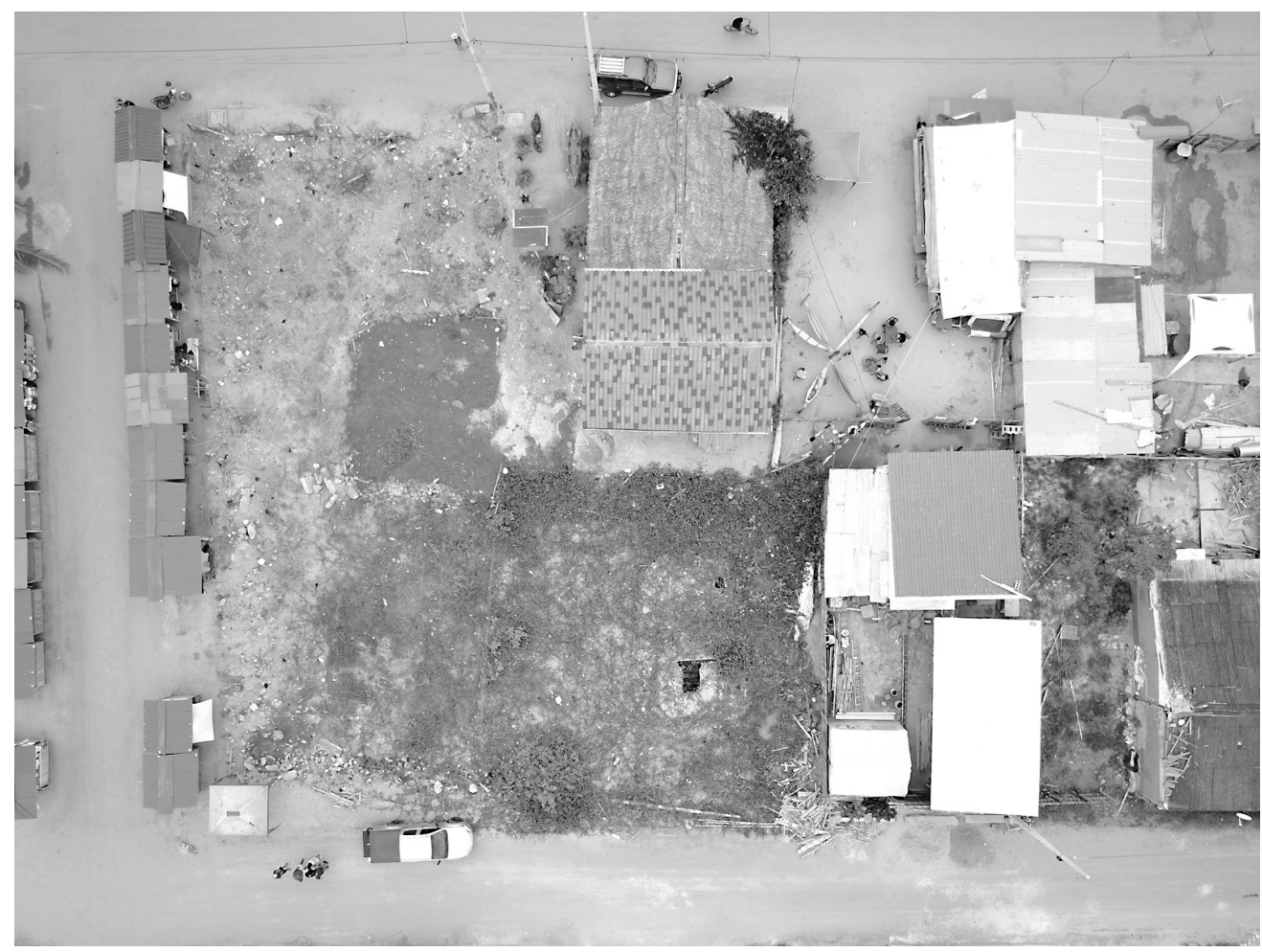

Figura 2: Vista aérea de los lotes baldíos donde existieron edificios y se derrumbaron a causa del terremoto. Estaban ubicados en la calle de artesanías, cerca al malecón de Canoa. Fotografía propia.

esta contenga ciertas irregularidades o no respondan a las necesidades reales de las personas:

...en otros casos mucha gente ha recibido ayuda, pero no real, por ejemplo, mi mami, a ella le dieron un certificado que dicen que le construyeron la casa, pero eso no pasó; a ella le arreglaron la casa, en su casa se cayeron dos paredes y eso lo arreglaron, sí pusieron dos paredes, pero nunca construyeron la casa. Le dieron un certificado de que sí recibió la casa. La casa cuesta 10 mil dólares, pero los arreglos no fueron ni cuatro mil. ${ }^{45}$

La lógica vertical incluye otras decisiones que están acordes con objetivos políticos del gobierno. El mejor ejemplo son las decisiones gubernamentales sobre la península Muisne de Esmeraldas después del terremoto. Esta parroquia tiene ciertas

45. (Entrevista 5). 
particularidades ${ }^{46}$ primero, por su carácter peninsular y, segundo, por la dependencia político-administrativa de la alcaldía ubicada en el continente, que le hizo más vulnerable al ejercicio de gubernamentalidad y biopolítica ejercida por el gobierno.

La isla de Muisne tuvo 256 heridos, 1.104 albergados, 312 casas destruidas, y 276 edificaciones afectadas. Los daños fueron mayores en las zonas aledañas o ubicadas en los esteros o manglares, además se afectaron los servicios básicos, telecomunicaciones y otras infraestructuras.

Ante este panorama, el gobierno a través de la Secretaria de Gestión de Riesgos emitió la Resolución No. SGR-73-2016 que declaró la isla como "zona de riesgo" por estar debajo de la zona de inundación y peligro de tsunami, indicando que para salvaguardar a la población esta debe ser reubicada en el continente. Sobre este punto hay polémica, pues pobladores de la península y activistas desacreditaron dicha decisión ya que estaría basada en un informe del 1 de junio de 2016, que se respaldó en una tesis de pregrado de $2004,{ }^{47}$ que, a su vez, copia datos sobre un tsunami de 1906 . No obstante, según estudios y modelos matemáticos del Instituto Oceanográfico de la Armada (INOCAR), las zonas de San Lorenzo, Muisne, Mompiche, Cojimíes y Boca de Briceño son zonas de muy alto riesgo de tsunamis. ${ }^{48}$

La mencionada resolución establece que la gobernación, prefectura y otras dependencias administrativas dispongan dicha reubicación, que se inició con el traslado de los centros gubernamentales, de salud, educación y otros servicios pocos meses después el terremoto. Posteriormente se prohibió que las ONG, organizaciones humanitarias y sociales y los organismos internacionales puedan prestar ayuda en la isla. Dichas decisiones buscaban obligar a la gente a dejar sus actividades, hogares y que, de a poco, se acojan a la ayuda del gobierno y se instalen en los albergues.

Muisne tiene aproximadamente doce mil habitantes, y la reubicación de todos ellos supondría la creación de una infraestructura grande, con capacidades que permitan albergar a dicha cantidad de personas, además de dotar de los medios de vida por un tiempo indeterminado. En otras palabras, este plan implicaría construir una ciudad en un breve lapso de tiempo.

46. Muisne es una península rodeada de manglares y estero del mar, lo que dificulta su accesibilidad, pues la única forma de acceso era a través de la lancha que cruce el río.

47. Mario Cruz DeHowitt, María Cristina Acosta y Nelson Eddy Vásquez, Riesgos por tsunami en la Costa ecuatoriana (Quito: ESPE, 2004). Disponible en http://www.ipgh.gob.ec/documentos/recursos/Riesgos\%20Tsunami\%20Costa\%20ecuatoriana.pdf . Consulta: 12 de diciembre de 2017.

48. Julio Granados, "Ubicación de un sistema de señalización frente a la amenaza de tsunamis para las playas de la provincia de Esmeraldas". Monografía de grado del Segundo Diplomado en Gestión Integral de Riesgos y Desastres (Quito: Instituto de Altos Estudios Nacionales, 2010). Disponible en «http://repositorio.iaen.edu.ec/bitstream/24000/437/1/IAEN-M019-2010.pdf . 
La reubicación, según el gobierno, sería a la zona de Bunche en el continente, donde se construyen mil viviendas, según información del Dirección Provincial de MIDUVI. Sin embargo, estas serían para los damnificados del terremoto, además que no cubrirían las necesidades de toda la población de Muisne: "No les exigimos que desalojen, pero deben hacerlo por su bien. Ahora el Estado no puede construir viviendas para que estén deshabitadas. Si dejan la isla, el gobierno puede construir, pero no lo vamos a hacer en vano". ${ }^{49}$

Obtener una casa del MIDUVI requiere, sin embargo, un copago de 10\% sobre los costos totales de la construcción. ${ }^{50}$ Se descontará automáticamente los montos faltantes del Bono del Desarrollo Humano en el caso de personas con demora en pago, lo que impacta directamente las capacidades reales de la gente afectada, además de disciplinarlas, según los planteamientos de la biopolítica en el sentido de Foucault.

Para las organizaciones sociales, las zonas del Relleno y Bunche también se encuentran en riesgo y son susceptibles de sufrir los estragos de un tsunami. Entonces el traslado no lograría dar seguridad a la población. Otro elemento que contradice los argumentos oficiales es que el gobierno tiene en efecto un plan y un proyecto eco-turístico para implementarse en la isla, como pudimos constatar en Dirección Provincial del MIDUVI. Este plan, parte del Programa Nacional de Destinos de Excelencia del Ministerio de Turismo ya desde el junio de $2016,{ }^{51}$ previene transformar la isla, pues dejaría de ser habitable y ser un gran complejo turístico donde se puedan visitar zonas protegidas y áreas recreacionales (parque acuático, zonas de restaurantes y otras):

En el caso de Muisne nosotros contratamos un proyecto turístico que esperamos se ejecute, no se obliga a nadie a reubicarse, eso es voluntario, les decimos que su actividad comercial no la dejen, pero sí que vivan en el continente, la escuela de los chicos, su vida, etc. La idea es que allá no se realice nada de vida familiar, solo actividad turística, avistamiento de tortugas, visitas a los manglares. Es un proyecto total que comprende toda la isla. La administración será municipal, nosotros le entregamos el proyecto, y ellos se encargan de financiar y ver la ejecución, nosotros esperamos que se realice. ${ }^{52}$

Es llamativo que para los casos de Canoa y Chamanga, ya que también existen proyectos turísticos previstos desde el MIDUVI, donde las familias de los actuales

49. Directora provincial MIDUVI.

50. Según la directora de la Subsecretaría de Vivienda en Quito, los costos de todas las construcciones (para cuatro familias hasta para una familia) son iguales: 10.000 dólares.

51. Elizabeth Bravo, "El sismo del 16 de abril en Manabí visto desde la ecología política del desastre", Universitas No. 26 (2017): 245. Disponible en 〈https://doi.org/http://doi.org/10.17163/uni.n26.2017.10〉.

52. Entrevista directora provincial del MIDUVI Esmeraldas, Esmeraldas, 3 de abril de 2017. 
pescadores artesanales podrán trabajar, paradójicamente solo durante el día, una vez que su reubicación bajo el pretexto de riesgo sea finalizada.

Los objetivos y políticas planteadas por el gobierno en el caso del reordenamiento de Chamanga, o el proyecto ecoturístico en Muisne, tienen un carácter unilateral, en el que no existe participación de la población; tampoco hubo procesos de consulta, según nuestros interlocutores, por lo que la ciudadanía es receptora al margen de estar de acuerdo, o si le resuelven los problemas o necesidades reales.

La declaratoria de emergencia permitió que el gobierno tome una serie de decisiones que fueron impuestas sobre la población. Las soluciones nunca contaron con la participación de la gente y muchas de las políticas no estuvieron enfocadas en la población sino en la reconstrucción física, lo que les obliga a cambiar la forma y los medios de vida de las personas, sin considerar sus afectos y dinámicas sociales:

Si la idea es de uno, esa idea es personal, entonces no es trabajo de la gente, y eso no siempre funciona, la gente debe participar... la ayuda es eso, debe ayudar a que la gente progrese, que la gente tenga, se sienta cómoda, para vivir mejor, por ejemplo, a las personas les dicen que renuncien a sus solares para ir a vivir a esas casas, pero que no son propias de uno. ${ }^{53}$

En el presente caso, la lógica top-down de la política implementada acrecentó las brechas sociales, económicas y políticas, pues mantiene o reproduce vulnerabilidades existentes ex ante, impidiendo que se consoliden y creen condiciones para el pleno cumplimiento de derechos de las poblaciones. Además la política de reactivación económica en las zonas de desastre, en lugar de construir resiliencia por medio de un desarrollo local, ${ }^{54}$ privilegia la entrega de créditos a negocios con gran capital (económico o inmueble) que pueden respaldar las deudas ante entidades bancarias.

Según nuestros diarios de campo, los créditos fueron otorgados a camaroneras cuyos dueños frecuentemente no son de la zona sino de ciudades como Guayaquil, Santo Domingo o Quito. Estos cuentan con amplio capital, lo que en el futuro mantendrá una estructura capitalista que ve en la población mano de obra dependiente de este tipo de actividades comerciales. Se trata además de un negocio que se expande en detrimento de la protección natural frente a tsunamis, es decir, los manglares o zonas protegidas (particularmente en Canoa y Muisne). En cambio, los pescadores artesanales y pequeños negocios (restaurantes, tiendas, hoteles, etc.), que eran los que mantenían la estructura y el dinamismo económico de las poblaciones afectadas, no recibieron la

53. Entrevista 5: damnificada, anónima por protección a la fuente, Chamanga, 3 de abril de 2017.

54. Ann-Margaret Esnard y Alka Sapat, Displaced by Disaster. Recovery and Resilience in a Globalizing World. 
atención o respaldo crediticio por no contar con garantías necesarias para sostener el pago de una deuda.

Los emprendimientos para mejorar las condiciones de vida de algunos damnificados tampoco son respaldados, pues la política bancaría requiere garantías de capital o algún bien inmueble, algo que no pueden cumplir la mayoría de afectados. Tradicionalmente, muchos agricultores o pescadores artesanales no tienen títulos de propiedad, ya que siempre tuvieron el derecho propietario, por lo que no hay forma de usar sus tierras o casas como garantía. En resumen, como ya se dijo, algunas políticas mantienen las desigualdades económicas, sociales y culturales que equivalían a un incumplimiento de algunos derechos humanos. Adicionalmente la verticalidad de la reconstrucción evita que las estructuras económicas cambien y exista una diversificación que responda a encadenamientos productivos distintos a los oligopólicos de grandes capitales (camaroneras, atuneras, monocultivos, u otros).

\section{CONSIDERACIONES FINALES}

Mediante algunas herramientas etnográficas, esta contribución ha revisado el proceso de la reconstrucción en espacios semiurbanos en Manabí y Esmeraldas. A pesar del carácter preliminar de nuestro estudio, el objetivo planteado fue ampliar las perspectivas fisicalistas que se presentan como dominantes hasta hoy día, unilateralmente y desde arriba hacia abajo. El enfoque ampliado es igualmente demandado por el marco legal y político vigente en el país y fue adicionalmente recién defendido a nivel internacional en el marco del último UPR del Comité de los DD. HH. Sin embargo, de manera interna, no se cumple con el mismo.

Desde tiempos coloniales Manabí y Esmeraldas se han caracterizado por limitaciones importantes en cuanto al respeto, protección y realización de los derechos civiles y políticos, así como derechos económicos, sociales y culturales. Ello por la existencia de grandes terratenientes, políticamente poderosos, economía clandestina, estructuras clientelistas, la degradación de importantes espacios ecológicos, entre otros. ${ }^{55}$ Adicionalmente, estas regiones siempre estuvieron al margen de la planificación estatal para la dotación de servicios básicos, seguridad, monitoreo de construcciones y, más ampliamente, del desarrollo sostenible local. Estas condiciones contribuyeron a la transformación de una aguda catástrofe natural en un verdadero desastre socioeconómico.

55. Carmen Dueñas de Anhalzer, Historia económica y social del norte de Manabi (Quito: Abya-Yala, 1986). 
Sin embargo, contrariamente a lo que subrayan guías y estándares internacionales de la reconstrucción como principios primordiales ${ }^{56}$ las decisiones políticas con respecto a la atención de los afectados fueron tomadas y ejecutadas sin la participación de organizaciones sociales o instituciones locales de las zonas afectadas, lo que dificulta no solo su implementación, sino la reconstrucción del tejido social y de las dinámicas económicas y políticas en las zonas afectadas.

La biopolítica ejercida por el gobierno restringió y vulneró de manera indirecta varios derechos humanos,${ }^{57}$ ya sea durante el "restablecimiento" de la vida normal, o en la administración de los albergues, donde de alguna manera, suprimieron capacidades y derechos, estableciendo un régimen militar de control, que impedía que los afectados puedan retomar de a poco con su vida. Son estas rupturas de la trayectoria individual que pueden ser muy impactantes en términos de capacidades e inclusive causar traumas permanentes, particularmente en los casos del desalojo forzado. ${ }^{58} \mathrm{Las}$ acciones gubernamentales, si bien dieron ayuda y asistencia, no fueron imparciales sino condicionadas por criterios y objetivos políticos, además de que se vulneraron los principios de protección.

Brindar respuestas sensibles a las condiciones de vida ante la catástrofe, alterándolas a largo plazo de manera sostenible y desde abajo hacia arriba, requería la creación y constante adaptación de estrategias de marcos de reconstrucción a través de la colaboración interinstitucional y con la sociedad civil. ${ }^{59}$

Para aterrizar estas herramientas y construir un marco tal desde las necesidades y derechos individuales, se sugiere tomar en cuenta el marco legal y político (supuestamente) vigente, los indicadores de DD. HH. y el enfoque del desarrollo humano. Estas perspectivas no solo permiten evitar en principio las fallas discutidas en este artículo, como, por ejemplo, las estrategias de biopolítica, la intervención incoherente, profusa y en contra de la sostenibilidad, sino también la limitación de capacidades a nivel

56. GFDRR et al., "Guide to Developing Disaster Recovery Frameworks. Sendai Conference Version”, en Jennifer E. Duyne Barenstein y Esther Leemann, edit., Post-Disaster Reconstruction and Change. Communities 'Perspectives (Boca Raton/London/New York: CRC Press/Taylor \& Francis, 2013).

57. Solo con vista a la Declaración Universal de los Derechos Humanos (1948) son directamente afectados ( vilidad (art. 13), a la expresión libre (art. 19), a estándares adecuados de vida (art. 25).

58. Jennifer Duyne Barenstein y Brigitte Marti Rojas Rivas, "Is Resettlement a Viable Strategy to Mitigate the Risk of Natural Disasters? Views and Voices from the Citizens of Santa Fe, Argentina", en Jennifer E. Duyne Barenstein y Esther Leemann, edit., Post-Disaster Reconstruction and Change: Communities' Perspectives.

59. Abhas K. Jha et al., Safer Homes, Stronger Communities: A Handbook for Reconstruction after Natural Disasters; GFDRR et al., "Guide to Developing Disaster Recovery Frameworks. Sendai Conference Version". 
local. Asimismo, permiten ampliar la protección ambiental y derechos al nivel local, lo que, a largo plazo, promoverá la confianza en las instituciones y en el Estado de derecho, al mismo tiempo que facilitaría la construcción de un marco apropiado para la prevención de desastres.

\section{BIBLIOGRAFÍA}

Alkire, Sabina. "Human Development: Definitions, Critiques, and Related Concepts". OPHI Working Papers, No. 36 (2010). 〈http://www.ophi.org.uk/human-development-definitionscritiques-and-related-concepts>.

Bass, S., R. Selvaraju, Dey de Pryck y F. Battista. Análisis de sistemas de gestión del riesgo de Desastres. s. 1.: Organización de las Naciones Unidas, 2009.

Bravo, Elizabeth. "El sismo del 16 de abril en Manabí visto desde la ecología política del desastre". Universitas, No. 26 (2017). Disponible en 〈https://doi.org/http://doi.org/10.17163/ uni.n26.2017.10\%.

Cobey, Ryan J., y M. L. Bandeira. "Sumak Kawsay or Buen Vivir, an alternative model in the Andean State?". Farol, Revista de Estudos Organizacionais e Sociedade, No. 3 (2010).

Consejo de Derechos Humanos. "Informe nacional presentado con arreglo al párrafo 5 del anexo de la resolución 16/21 del Consejo de Derechos Humanos-Ecuador". Ginebra, 4 de julio de 2017.

Cruz DeHowitt, Mario A., María Cristina Acosta y Nelson Eddy Vásquez. Riesgos por tsunami en la Costa ecuatoriana. Quito: Escuela Politécnica del Ejercito, s. f.

De Béco, Gauthier. "Human rights indicators and MDG indicators: building a common language for human rights and development organizations". En Paul Gready y Wouter Vandenhole, edit., Human Rights and Development in the New Millenium. Oxon/New York: Routledge, 2014.

Deneulin, Séverine. "Beyond Individual Freedom and Agency: Structures of Living together in the Capability Approach". En Flavio Comim, Mozaffar Qizilbash y Sabina Alkire, edit., The Capability Approach: Concepts, Measures and Applications. Cambridge: Cambridge University Press, 2008.

—, y Lila Shahani, edit. An Introduction to the Human Development and Capability Approach: Freedom and Agency. London: Earthscan, 2010.

Dueñas de Anhalzer, Carmen. Historia económica y social del norte de Manabí. Quito: AbyaYala, 1986.

Duyne Barenstein, Jennifer E., y Brigitte Marti Rojas Rivas. "Is Resettlement a Viable Strategy to Mitigate the Risk of Natural Disasters? Views and Voices from the Citizens of Santa Fe, Argentina". En Jennifer E. Duyne Barenstein y Esther Leemann, edit., Post-Disaster Reconstruction and Change: Communities' Perspectives. Boca Raton/London/New York: CRC Press/Taylor \& Francis, 2013. 
-, y Esther Leemann, edit. Post-Disaster Reconstruction and Change. Communities' Perspectives. Boca Raton/London/New York: CRC Press/Taylor \& Francis, 2013.

Ecuador. Constitución Política de la República. Montecristi, 2008.

Esnard, Ann-Margaret, y Alka Sapat. Displaced by Disaster. Recovery and Resilience in a Globalizing World. Nueva York/Oxon: Routledge, Taylor \& Francis, 2014.

Foucault, Michel. Nacimiento de la biopolítica. Curso del Collège de France (1978-1979) Madrid: Akal, 2009.

GFDRR, et al. "Guide to Developing Disaster Recovery Frameworks. Sendai Conference Version”. En Disaster Recovery Framework. Washington D. C.: Global Facility for Disaster Reduction and Recovery/Banco Mundial/PNUD/Unión Europea, 2015.

Haque, C. Emdad, y David Etkin, edit. Disaster Risk and Vulnerability. Mitigation through Mobilizing Communities and Partnerships. Montreal/Kingston/London/Ithaca: McGillQueen's University Press, 2012.

INEC. Reconstruyendo las cifras luego del sismo. Memorias. Quito: Instituto Nacional de Estadística y Censos, 2017.

Jha, Abhas K., Jennifer Duyne Barenstein, Priscilla M. Phelps, Daniel Pittet, y Stephen Sena. Safer Homes, Stronger Communities: A Handbook for Reconstruction after Natural Disasters. Washington D. C.: The International Bank for Reconstruction and Development/The World Bank, 2010.

Lavell Thomas, Allan. "Ciencias sociales y desastres naturales en América Latina: un encuentro inconcluso". Revista Mexicana de Sociología, No. 2 (abril-junio 1993).

Marchezini, Víctor. "La producción silenciada de los desastres naturales en catástrofes sociales”. Revista Mexicana de Sociología, No. 2 (2014).

Nussbaum, Martha. Creating Capabilities: The Human Development Approach. Cambridge: Belknap Press of Harvard University Press, 2011.

-.Frontiers of Justice; Disability, Nationality, Species Membership. Cambridge: Harvard University Press, 2006.

Overseas Development Institute. The future framework for disaster risk reduction. A guide for decision-makers. Londres: ODI, 2014.

Pallares, Martín. "El Comité de Reconstrucción... es el mismo gobierno". Cuatro Pelagatos (blog). 30 de abril de 2016. Disponible en 〈http://4pelagatos.com/2016/04/30/el-comitede-reconstruccion-es-el-mismo-gobierno/ $>$.

Proyecto Esfera. Manual del proyecto Esfera. Carta Humanitaria y normas mínimas para la respuesta humanitaria. s. 1.: Proyecto Esfera, 2011.

Riedel, Eibe, Gilles Giacca y Christophe Golay. "The Development of Economic, Social, and Cultural Rights in International Law: Introduction". En Eibe Riedel, Giacca, Gilles, y Christophe Golay, edit., Economic, Social, and Cultural Rights in International Law. Oxford: Oxford University Press, 2014. 
Rodríguez Velásquez, Daniel. La política pública frente a desastres en el contexto de la reforma del Estado: opciones desde la sociedad civil. Políticas públicas y desastres. México, D. F.: Cooperación Internacional, 2008.

Sen, Amartya. Development as Freedom. Oxford/Nueva York: Oxford University Press, 1999.

SENPLADES (Secretaría Nacional de Planificación y Desarrollo). Buen Vivir Plan Nacional 2013-2017. Quito: SENPLADES, 2013.

-. Plan Nacional de Desarrollo. Plan Nacional para el Buen Vivir 2009-2013. Construyendo un Estado plurinacional e intercultural (Versión resumida). Quito: SENPLADES, 2009.

UN ACNUDH. Construcción de Indicadores de Derechos Humanos: Experiencias regionales. México: UNHCHR, 2013.

—. "Informe de misión. Asistencia técnica al proyecto SIDERECHOS. Septiembre 2010". Quito, 2010. Private copy; with permission of the authors.

UN OHCHR. Human Rights Indicators: A Guide to Measurement and Implementation. New York and Geneva: UN OHCHR, 2012.

-. "Report on Indicators for Promoting and Monitoring the Implementation of Human Rights". International Human Rights Instruments. Ginebra: UN OHCHR, 2008. Disponible en 〈http://www2.ohchr.org/english/bodies/icm-mc/docs/HRI.MC.2008.3EN.pdf〉.

Waldmüller, Johannes M. “Human Rights Indicators as 'Development 2.0’?’. Alternautas, No. 1 (2014).

- “ ' 'Living Well Rather Than Living Better' Measuring Biocentric Human-Nature Rights and Human-Nature Development in Ecuador". Journal of Social Quality, No. 2 (2015). Disponible en 〈https://doi.org/10.3167/IJSQ.2015.050202〉.

Walsh, Catherine. "The (Re)articulation of Political Subjectivities and Colonial Difference in Ecuador: Reflections on Capitalism and the Geopolitics of Knowledge". Neplanta: Views from South, No. 1 (2002).

\section{Entrevistas}

Entrevista 1: Damnificada viviendo en el albergue 1 de Muisne. Anónima por protección a la fuente, Muisne, 2 de abril de 2017.

Entrevista 2: Damnificada, anónima por protección a la fuente, Chamanga, 3 de abril de 2017.

Entrevista 3: Directora provincial del MIDUVI Esmeraldas, Esmeraldas, 3 de abril de 2017.

Entrevista 4: Presidente del GAD Parroquial de Canoa, Canoa, 2 de junio de 2017.

Entrevista 5: Damnificada, anónima por protección a la fuente, Chamanga, 3 de abril de 2017. 